\title{
Morbidity and medicine prescriptions in a nationwide Danish population of patients diagnosed with polycystic ovary syndrome
}

\author{
Dorte Glintborg, Katrine Hass Rubin ${ }^{1}$, Mads Nybo², Bo Abrahamsen ${ }^{1}$ and \\ Marianne Andersen
}

Department of Endocrinology, Odense University Hospital, Kløvervænget 6, 3rd floor, 5000 Odense C, Denmark, ${ }^{1}$ OPEN - Odense Patient data Explorative Network, Institute of Clinical Research, University of Southern Denmark, Odense, Denmark and ${ }^{2}$ Department of Clinical Biochemistry and Pharmacology, Odense University Hospital, Odense, Denmark

\author{
Correspondence \\ should be addressed \\ to D Glintborg \\ Email \\ dorte.glintborg@rsyd.dk
}

\begin{abstract}
Objective: The prevalence of type 2 diabetes is increased in polycystic ovary syndrome (PCOS), but the prevalence of other diseases is not clarified. We aimed to investigate morbidity and medicine prescriptions in PCOS.

Design: A National Register-based study.

Methods: Patients with PCOS (PCOS Denmark and an embedded cohort; PCOS Odense University Hospital (OUH)) and one control population. Premenopausal women with PCOS underwent clinical and biochemical examination (PCOS OUH, $n=1217)$. PCOS Denmark ( $n=19$ 199) included women with PCOS in the Danish National Patient Register. Three age-matched controls were included per patient $(n=57483)$.

Main outcome measures: Diagnosis codes and filled prescriptions.

Results: The mean (range) age of the PCOS Denmark group and controls was 30.6 (12-60) years. Patients in PCOS Denmark had higher Charlson index, higher prevalence of diabetes, dyslipidemia, and hypertension than controls. PCOS was associated with a two times increased risk of stroke and thrombosis, whereas the risk of other cardiovascular diseases was not increased. Thyroid disease, asthma, migraine, and depression were more prevalent in PCOS Denmark vs controls, whereas fractures were rarer. Infertility was increased in patients compared with controls, but the mean number of births was higher in PCOS. Medicine prescriptions within all diagnosis areas were significantly higher in PCOS patients than in controls. In PCOS OUH, polycystic ovaries (PCO) and irregular menses were associated with a more adverse metabolic risk profile, but individual Rotterdam criteria were not associated with cardiometabolic diagnoses.

Conclusion: Cardiometabolic and psychiatric morbidity were significantly increased in a Danish population with PCOS. Medical diseases are frequent also in young patients with PCOS.

European Journal of

Endocrinology

(2015) 172, 627-638
\end{abstract}

\section{Introduction}

Polycystic ovary syndrome (PCOS) is a prevalent endocrine condition, which is nowadays most often defined according to the Rotterdam criteria $(1,2)$. Importantly, PCOS is a diagnosis of exclusion and the Rotterdam criteria include irregular ovulation, biochemical/clinical hyperandrogenism, and/or polycystic ovaries (PCO) (1). In women with hirsutism, the prevalence of PCOS is more than $75 \%(3,4)$. PCOS is characterized by subclinical inflammation and insulin resistance, which along with obesity may be associated with increased morbidity (3). Hyperandrogenism and hyperandrogenemia in PCOS may further increase the metabolic risk $(5,6)$. Nearly $50 \%$ of patients with PCOS fulfill the criteria of the metabolic syndrome and PCOS is associated with a five to ten times

Published by Bioscientifica Ltd. 
increased risk of diabetes and thus increased number of cardiovascular risk factors $(7,8,9)$. Retrospective studies reported significantly higher frequency of hypertension and cardiovascular disease in PCOS, especially in women with irregular cycles $(10,11,12,13)$. Recommendations for the diagnosis and follow-up of patients with PCOS include screening for the metabolic syndrome and other cardiovascular risk factors with regular intervals $(3,14)$. These recommendations are based on clinical studies, whereas no register-based studies evaluated the prevalence of diabetes and cardiovascular disease in patients diagnosed with PCOS.

Hyper-inflammation in PCOS could affect the autoimmune function (15) and in small clinical studies the prevalence of autoimmune thyroiditis was increased (16). Furthermore, respiratory function in patients with asthma was associated with menstrual pattern (17) and PCOS could therefore be associated with changed respiratory health (17). These hypotheses could be further tested by studying the prevalence of thyroid and asthma diagnoses in populations with PCOS along with the medicine prescription pattern of thyroid hormones, anti-thyroid medicine, and asthma medicine.

Quality of life is impaired in patients with PCOS and the risk of depression is approximately eight times increased compared with controls $(18,19)$. PCOS could therefore be associated with increased use of antidepressants, analgesics, and neuropsychiatric medicine, but few studies evaluated this hypothesis. We recently reported that $20 \%$ of reproductive-aged patients with PCOS from our uptake area had prescriptions of antidepressants during follow-up compared with $15 \%$ healthy controls (20). However, to our knowledge, no population-based study investigated the prevalence of psychiatric and neurological diagnoses in patients with PCOS.

The aim of the present register-based study was to investigate possible associations between the diagnosis of PCOS and morbidity evaluated by diagnosis codes in the Danish National Patient Register (NPR) and medical prescriptions from the National Prescriptions Registry. Possible associations between individual Rotterdam criteria and cardiometabolic health were investigated in a well-described subgroup of patients with hyperandrogenism and/or PCOS.

\section{Patients and methods}

The study was designed as a register-based study including two patient populations with PCOS and a control population.

\section{Population 1, PCOS Odense University Hospital}

This embedded cohort included premenopausal women referred to the outpatient clinic at the Department of Endocrinology and Metabolism, Odense University Hospital (OUH) during 1997-2012 with the diagnoses of hirsutism (L680) and/or PCOS (E282).

\section{Inclusion criteria}

Women were included if they fulfilled the Rotterdam criteria for PCOS or had clinical and/or biochemical hyperandrogenism $(21,22,23)$.

\section{Exclusion criteria}

Women younger than 15 years were referred to the Department of Pediatrics at Odense University Hospital and patients with a primary complaint of infertility were referred to the local Fertility Clinic. Patients with serious endocrine diseases were excluded as previously described (8). Patients not attending for the measurement of hormonal or metabolic parameters were excluded.

Three patients diagnosed with PCOS before the age of 15 were included in the study as they underwent full evaluation at our department after the age of 15 years. Patients paused oral contraceptives for at least 3 months before evaluation.

\section{Methods}

Routine evaluation included medical history, clinical examination, transvaginal ultrasound, and fasting blood samples (8). Patients with regular periods and cycle length of 27-35 days were classified as having regular menses. The remaining patients were classified as anovulatory. Hirsutism was assessed by the Ferriman Gallwey score (FG-score) (24). Waist circumference was measured to the nearest centimeter in a standing position midway between the lower costal margin and the iliac crest.

Fasting blood samples were drawn in follicular phase (cycle days 2-8) in patients with a cycle length shorter than 3 months, while patients with cycle length $>3$ months had the blood samples drawn on a random cycle day. Blood tests included androgens (total testosterone, freetestosterone, sex hormone-binding globulin (SHBG), dehydroepiandrostenedione sulfate, 17-hydroxyprogesterone), LH, FSH, prolactin, HbA1c, lipid profile insulin, and blood glucose. 


\section{Assays}

Serum total testosterone and SHBG were analyzed using a specific RIA after extraction as previously described (25). The method has a close correlation with the determination of testosterone levels by mass spectrometry. The intra-assay coefficient of variation (CV) for total testosterone was 8.2 and $5.2 \%$ for SHBG. The inter-assay CV for total testosterone was 13.8 and $7.5 \%$ for SHBG. Free testosterone levels were calculated from measurements of total testosterone and SHBG. Insulin was analyzed by a time-resolved fluoroimmunoassay using a commercial kit (AutoDelfia, Wallac Oy, Turku, Finland) with an intraassay variation $2.1-3.7 \%$ and inter-assay variation $3.4-$ $4.0 \%$. Reference intervals for the individual hormones were as follows: total testosterone $0.55-1.8 \mathrm{nmol} / \mathrm{l}$, free testosterone $0.006-0.034 \mathrm{nmol} / \mathrm{l}$, SHBG $41-170 \mathrm{nmol} / \mathrm{l}$, and insulin $18-173 \mathrm{pmol} / \mathrm{l}$.

Plasma total cholesterol, HDL cholesterol, and triglycerides (TG) were analyzed by enzymatic colorimetric reactions (Modular P, Roche), while LDL cholesterol was calculated using the Friedewald equation. Blood glucose was measured on capillary ear blood using Hemo Cue. We calculated HOMA- $\mathrm{R}=$ fasting insulin $\times$ fasting blood glucose/22.5 (26), reference $\leq 15$ pmol $\mathrm{mmol}^{-2}$.

\section{Population 2, PCOS Denmark}

Population 2 included patients diagnosed with hirsutism or PCOS according to the International Classification of Diseases (ICD) 10 (L680 and E282) in the Danish NPR from 1995 to 2012 over the age of 12 years. The Danish NPR contains discharge diagnosis codes according to ICD8 during 1977-1993 and ICD10 from 1994 and onwards. From 1977 to 1994 only inpatient contacts were registered, while from 1994 both in- and outpatient contacts are registered (27). The current sub-classification of diabetes mellitus types was not used until 1994, as the ICD8 coding classified diabetes mellitus patients as having insulindependent diabetes mellitus (ICD8 code 249) or noninsulin-dependent diabetes mellitus (ICD8 code 250). Hence, the GDM diagnosis did not exist in the ICD8 coding and a decision was made to include data from 1994 and onwards in this study. PCOS can only be diagnosed after menarche (28) and therefore only patients aged 12 years or more were included in the PCOS Denmark study population (29). The index year was defined as the first time a patient received the diagnosis L680 or E282. The PCOS OUH population was a subset of the PCOS Denmark population.

\section{Controls}

For each patient in the PCOS Denmark population three controls were randomly drawn from the NPR. The controls were born in the same year as PCOS cases and had to be alive on the index date of their respective PCOS case. Controls were assigned the index date of their matched PCOS cases.

\section{Exclusion diagnoses}

The PCOS diagnosis requires exclusion of diagnoses that may cause symptoms similar to the condition (1). We excluded subjects in both study arms with a history of the following conditions: E221 (hyperprolactinemia), E220 (acromegaly), E24 (Cushing's disease), E25 (adrenogenital syndrome), and Q96 (Turner syndrome).

\section{The Danish Health Registries}

All individuals in Denmark are assigned with a unique personal identification number and data from all public registers can be linked at an individual level. All ICD10 hospital diagnoses were extracted from the NPR on the total study population. Information on all prescriptions issued by prescribers, general practitioners, or specialists in Denmark from 1995 to 2012 were retrieved from the National Prescriptions Registry. The National Prescriptions Registry database contains a complete record of Anatomic Therapeutic Chemical code, date drug dispensed, number of drug packets, and number of tablets per packet for all prescriptions filled at Danish pharmacies. The National Cause of Death Register contains information on all deaths. It includes death certificate diagnoses and date of death.

The core study was an open register-based cohort study. The present paper is a description of baseline characteristics preceding the first diagnosis of PCOS (the index date). Specifically, for comorbid conditions, we included hospital contacts since 1995. The Charlson Comorbidity Index is based on 19 comorbid conditions (30) and was calculated from the ICD10 operationalization by Quan et al. (31). For prescriptions, we made a distinction between recent use (at least one prescription of the drug in question in the last 12 months before the index date) and ever use (at least one prescription since establishment of the register in 1995 to the index date). The study did not need approval from the local Ethics committee or Institutional Review Board by Danish law. Transfer of patient data from the PCOS OUH cohort to Statistics Denmark (project no 704175) for merging with national datasets was approved by the Data Protection Agency. 


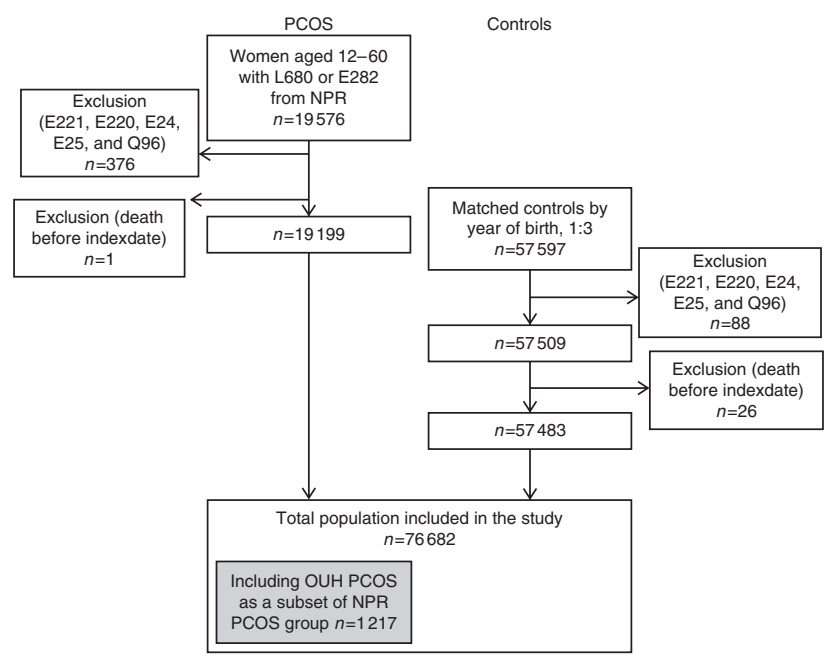

Figure 1

Flowchart of included subjects.

\section{Statistical analyses}

The statistical approach addressed two main questions: i) did the patients in PCOS OUH differ from patients in PCOS Denmark on key characteristics and ii) did the patients in PCOS Denmark differ from random population controls. Descriptive analyses for categorical variables (e.g., diabetes, thyroid disease) were presented as frequencies and evaluated by $\chi^{2}$ tests. Continuous variables (e.g., number of births) were tabulated as medians (with quartiles) or means (with s.D.) depending on their distribution. Mann-Whitney $U$ tests were used to test for differences between the groups for these variables. $P$ values below 0.05 were considered to be statistically significant. All analyses were conducted using STATA 13 through a remote VPN access to Statistics Denmark and the analysts were blinded to the personal identities of the study subjects.

\section{Results}

The flow chart of included women is summarized in Fig. 1.

\section{PCOS OUH ( $n=1217)$ : clinical, biochemical data, and individual Rotterdam criteria}

Clinical and biochemical data from the PCOS OUH cohort are presented in Table 1 . Patients with PCO $(n=1085)$ were younger and had higher BMI, waist, T-testosterone, fasting BG, fasting insulin, and HOMA-R levels compared with patients with normal US, whereas FG-score was lower (Table 2).
The majority of patients had biochemical and/or clinical hyperandrogenism $(1124 / 1211=92.8 \%$, Table 2$)$. Measures of body composition and metabolic risk profile were comparable in patients with and without hyperandrogenism.

Patients with irregular menses were younger and had higher BMI, waist, total- and free testosterone, TG, fasting BG, fasting insulin, and HOMA-R levels compared with patients with regular menses, whereas FG-score, SHBG, and HDL were lower (Table 2).

\section{Characteristics and diagnoses in patients with PCOS and controls ( $n=76$ 682)}

PCOS Denmark ( $n=19$ 199) vs controls $(n=57483)$ - The mean age of the PCOS Denmark group and controls were 30.6 years. The Charlson Index was higher in patients with PCOS compared with controls. The PCOS Denmark group had higher prevalence of the diagnoses obesity, all types of diabetes, dyslipidemia, hypertension, stroke, and thrombosis compared with controls, whereas the risk of cardiovascular disease, myocardial infarction, and transitory cerebral ischemia was not increased in PCOS. Furthermore, patients with PCOS had increased prevalence of all types of thyroid disease, asthma, depression, migraine, cholecystitis, and malignant diseases compared with controls, whereas fractures were found in fewer patients. The prevalence of infertility was higher in PCOS compared with controls, but the mean number of births was higher in PCOS and the distribution of number of births differed significantly between patients and controls.

Table 1 Clinical and biochemical data from the PCOS OUH patient cohort ( $n=1217)$. Data presented as median (quartiles).

\begin{tabular}{|c|c|c|}
\hline & $\boldsymbol{n}$ & Median (quartiles) \\
\hline Age (years) & 1217 & $29(23-35)$ \\
\hline BMI $\left(\mathrm{kg} / \mathrm{m}^{2}\right)$ & 1130 & $27.3(23.0-32.7)$ \\
\hline Waist (cm) & 766 & $89(78-104)$ \\
\hline FG total & 1069 & $11(5-15)$ \\
\hline T-testosterone (nmol/l) & 866 & $1.74(1.24-2.38)$ \\
\hline SHBG (nmol/l) & 1126 & $44(31-66)$ \\
\hline Free testosterone ( $\mathrm{nmol} / \mathrm{l})$ & 852 & $0.033(0.021-0.050)$ \\
\hline Cholesterol (mmol/l) & 906 & $4.6(4.1-5.3)$ \\
\hline TG $(\mathrm{mmol} / \mathrm{l})$ & 894 & $1.0(0.7-1.5)$ \\
\hline HDL (mmol/l) & 895 & $1.4(1.1-1.6)$ \\
\hline LDL (mmol/l) & 892 & $2.7(2.2-3.3)$ \\
\hline Fasting blood glucose $(\mathrm{mmol} / \mathrm{l})$ & 550 & $4.6(4.3-5.0)$ \\
\hline Fasting insulin (pmol/l) & 588 & $56(38-91)$ \\
\hline HOMA-R (pmol mmol l-2) & 557 & $12.2(8.1-20.1)$ \\
\hline
\end{tabular}



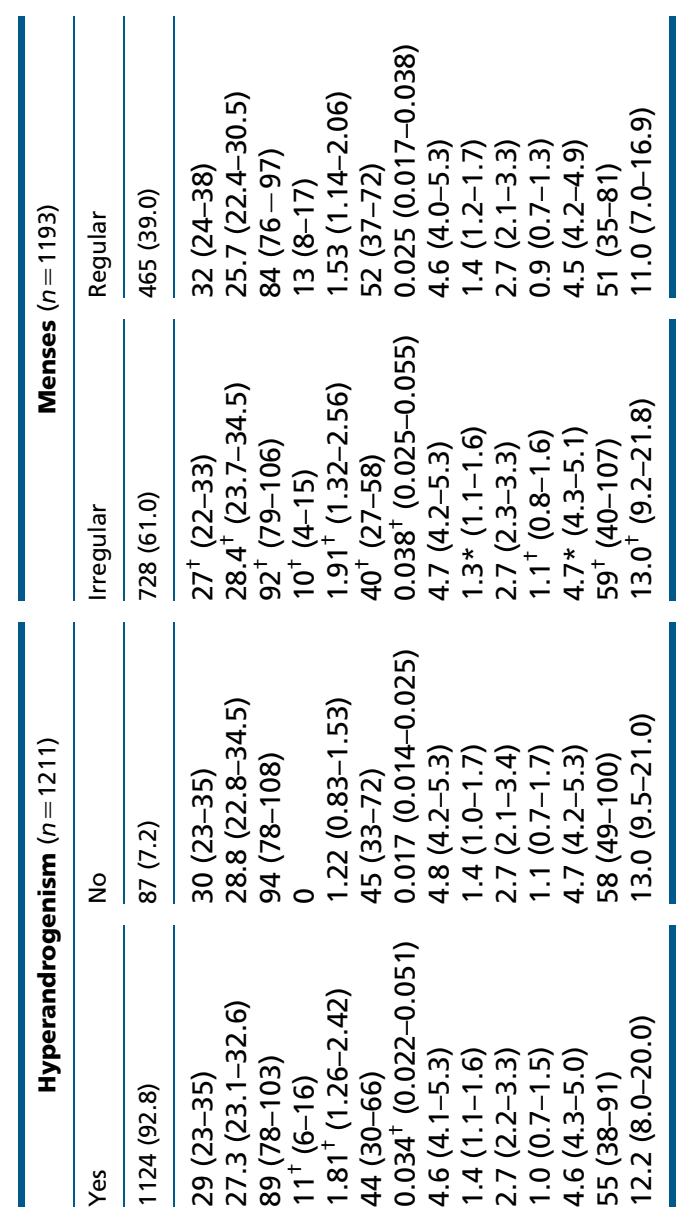

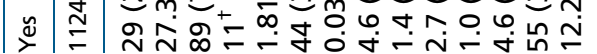
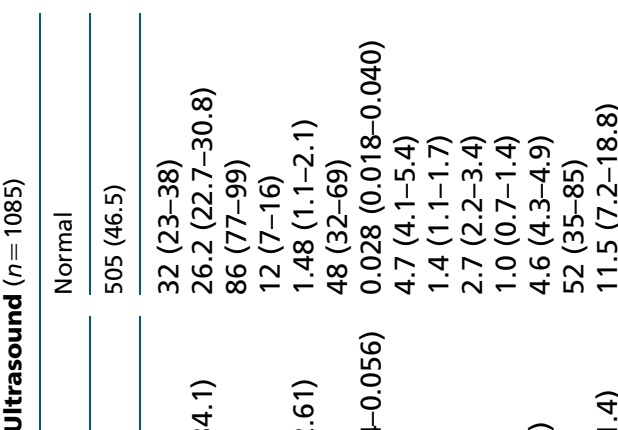

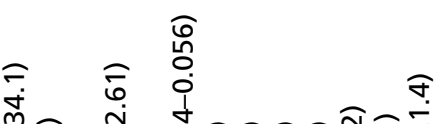

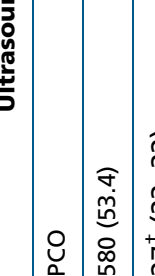

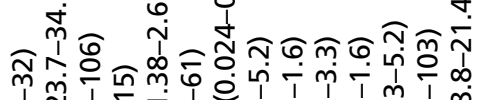

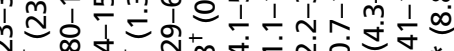

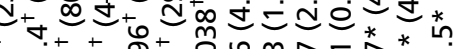

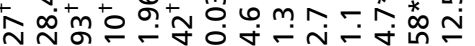

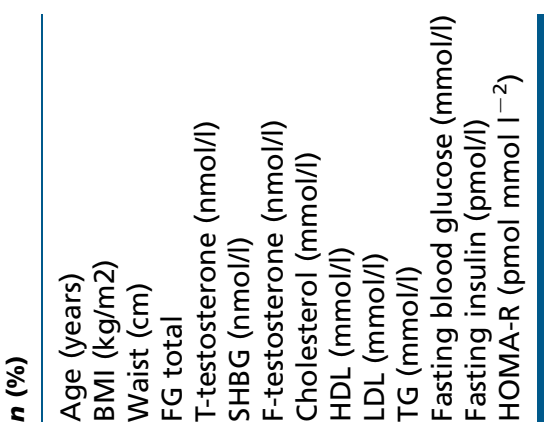

PCOS OUH $(n=1217) \quad$ vs PCOS Denmark $(n=19199)$ The PCOS OUH group had lower mean age (29.3 years) than patients in PCOS Denmark (Table 3). Patients in the PCOS OUH group had lower prevalence of migraine, malignant disease and infertility compared with the PCOS Denmark group. Otherwise, the prevalence of diagnoses was comparable. Patients in PCOS OUH had lower mean number of births compared with PCOS Denmark and the distribution of number of births differed between the two patient groups.

Medical treatment in patients with PCOS and controls $(n=76682) \triangleright$ Table 4 gives all time use of medical treatment in the study population.

PCOS Denmark $(n=19$ 199) vs controls $(n=57483)$ Patients with PCOS had higher use of all types of antidiabetic treatment, anti-lipids, antihypertensives, allergy medicine, asthma medicine, antidepressants, neuroleptics, anxiety medicine, analgesics, thyroid, anti-thyroid treatment, antibiotic treatment, all types of oral contraceptives, gestagen, intrauterine devices, antiandrogen treatment, and fertility treatment compared with controls.

PCOS OUH $(n=1217) \quad$ vs PCOS Denmark $(n=19199)$ The prescription of metformin, drospirenone/ethinylestradiol, and fertility treatment was lower in PCOS OUH vs PCOS Denmark, whereas prescriptions of antibiotic treatment and cyproterone acetate/ethinylestradiol were higher.

Table 5 gives use of medical treatment 1 year before the diagnosis of PCOS.

PCOS Denmark $(n=19$ 199) vs controls $(n=57483)$ Patients with PCOS had higher use of metformin, insulin, other anti-diabetics, anti-lipids, antihypertensives, allergy medicine, asthma medicine, antidepressants, neuroleptics, anxiety medicine, analgesics, thyroid and anti-thyroid treatment, antibiotic treatment, oral contraceptives, 3rd generation oral contraceptives, drospirenone/ ethinylestradiol, cyproterone acetate/ethinylestradiol, gestagen, intrauterine devices, spironolactone, vaniqa, and fertility treatment compared with controls. Prescriptions of smoke stop, 2nd generation oral contraceptives, and finasteride were comparable between patients and controls.

PCOS OUH $(n=1217)$ vs PCOS Denmark $(n=19199)$ • Prescriptions for metformin, other anti-diabetics, 
Table 3 Characteristics of PCOS patients and controls. Data presented as $n$ (\%) or mean (s.D.) or min-max.

\begin{tabular}{|c|c|c|c|c|c|c|c|c|c|}
\hline & & & & & $\begin{array}{c}\text { PCOS Der } \\
(n=19\end{array}$ & & $\begin{array}{l}\text { Contr } \\
(n=57\end{array}$ & & \\
\hline & & $n$ & $\%$ & $\boldsymbol{P}^{\mathrm{a}}$ & $n$ & $\%$ & $n$ & $\%$ & $\boldsymbol{P}^{\mathrm{b}}$ \\
\hline $\begin{array}{l}\text { Age (years) at first diagnosis, } \\
\text { mean (s.D.) and min-max }\end{array}$ & & & & $<0.001$ & $\begin{array}{r}30.6(c \\
12-6\end{array}$ & & $\begin{array}{r}30.6(5 \\
12-6\end{array}$ & & 0.96 \\
\hline Charlson index, mean (s.D.) & & 0.11 & 447) & 0.062 & $0.155(0$ & & $0.096(0$ & & $<0.001$ \\
\hline Cardio-metabolic diseases & ICD10 codes & & & & & & & & \\
\hline Obesity & E66 & 167 & 13.7 & 0.07 & 2306 & 12.0 & 791 & 1.4 & $<0.001$ \\
\hline Diabetes & E10, E11, E13, E14 & 29 & 2.4 & 0.70 & 429 & 2.2 & 423 & 0.7 & $<0.001$ \\
\hline Type 1 diabetes & E10 & 7 & 0.6 & 0.29 & 166 & 0.9 & 307 & 0.5 & $<0.001$ \\
\hline Type 2 diabetes & E11 & 23 & 1.8 & 0.42 & 306 & 1.6 & 175 & 0.3 & $<0.001$ \\
\hline Gestational diabetes & O24, P70 & 18 & 1.5 & 0.94 & 289 & 1.5 & 217 & 0.4 & $<0.001$ \\
\hline Dyslipidemia & E78 & 5 & 0.4 & 0.50 & 107 & 0.6 & 108 & 0.2 & $<0.001$ \\
\hline Hypertension & 1109 & 14 & 1.2 & 0.10 & 343 & 1.8 & 365 & 0.6 & $<0.001$ \\
\hline Cardiovascular disease & 120,150 & 2 & 0.2 & 0.24 & 72 & 0.3 & 177 & 0.3 & 0.16 \\
\hline Mocardial infarction & $121,122,123,124,125$ & 2 & 0.2 & 0.76 & 39 & 0.2 & 121 & 0.2 & 0.85 \\
\hline Transitory cerebral ischemia & G45 & 2 & 0.2 & 0.75 & 25 & 0.1 & 52 & 0.1 & 0.13 \\
\hline Stroke & 163,164 & 2 & 0.2 & 0.54 & 49 & 0.3 & 82 & 0.1 & 0.001 \\
\hline Thrombosis, lung embolism & $126,0082,180,181,182$ & 6 & 0.5 & 0.19 & 127 & 0.7 & 183 & 0.3 & $<0.001$ \\
\hline Other diseases & & & & & & & & & \\
\hline Thyroid disease & E04, E05, E03 & 35 & 2.9 & 0.49 & 476 & 2.5 & 379 & 0.7 & $<0.001$ \\
\hline Atoxic goitre & E04 & 15 & 1.2 & 0.15 & 161 & 0.8 & 146 & 0.3 & $<0.001$ \\
\hline Hyperthyroidism & E05 & 9 & 0.7 & 0.87 & 150 & 0.8 & 159 & 0.3 & $<0.001$ \\
\hline Hypothyroidism & E03 & 15 & 1.2 & 0.69 & 213 & 1.1 & 106 & 0.2 & $<0.001$ \\
\hline Asthma & J44, J45 & 39 & 3.2 & 0.76 & 586 & 3.0 & 1270 & 2.2 & $<0.001$ \\
\hline Depression & F3 & 13 & 1.1 & 0.36 & 266 & 1.4 & 284 & 0.5 & $<0.001$ \\
\hline Migraine/headache & G43, G44 & 12 & 1.0 & 0.008 & 398 & 2.1 & 539 & 0.9 & $<0.001$ \\
\hline Fractures & S02-S92 & 138 & 11.3 & 0.11 & 2481 & 12.9 & 9280 & 16.1 & $<0.001$ \\
\hline Cholecystitis & $\mathrm{K} 8$ & 40 & 3.3 & 0.97 & 628 & 3.3 & 613 & 1.1 & $<0.001$ \\
\hline Malignant diseases & C00-D48 & 57 & 4.7 & 0.001 & 1368 & 7.1 & 2117 & 3.7 & $<0.001$ \\
\hline Fertility & & & & & & & & & \\
\hline Infertility & N97, Z35 & 207 & 17.0 & $<0.001$ & 4431 & 23.1 & 2337 & 4.1 & $<0.001$ \\
\hline Number of births, mean (s.D.) & & & 57) & 0.001 & $0.28(0$ & & $0.20(0$ & & $<0.001$ \\
\hline Number of births & 0 & 936 & 76.9 & 0.018 & 15447 & 80.5 & 49946 & 86.9 & $<0.001$ \\
\hline & 1 & 179 & 14.7 & & 2446 & 12.7 & 4403 & 7.7 & \\
\hline & 2 & 87 & 7.2 & & 1077 & 5.6 & 2561 & 4.5 & \\
\hline & $\geq 3$ & 15 & 1.2 & & 229 & 1.2 & 573 & 1.0 & \\
\hline
\end{tabular}

$P^{\mathrm{a}}$ Between PCOS OUH and PCOS Denmark, $P^{\mathrm{b}}$ Between PCOS Denmark and controls, $\chi^{2}$ test (for prevalences) and Mann-Whitney $U$ tests (for continuous variables).

antihypertensives, gestagen, and fertility treatment were lower in PCOS OUH than in PCOS Denmark, whereas prescriptions of intrauterine devices were higher.

\section{Rotterdam criteria in the PCOS OUH population and cardiometabolic diagnoses}

The presence of PCO, hyperandrogenism, or irregular menses was associated with obesity (Table 6). Six women in the study population had a diagnosis of thrombosis. The prevalence of thrombosis was significantly lower in patients with hyperandrogenism $(4 / 1124=0.4 \%)$ compared with patients without hyperandrogenism $(2 / 87=2.3 \%)$. Five of six patients diagnosed with thrombosis had been treated with oral contraceptives (3rd generation $n=4$, drospirenone/ethinylestradiol $n=1$ ).

\section{Discussion}

To our knowledge, this is the first nationwide, populationbased study that reports increased morbidity in patients with PCOS compared with healthy age-matched controls. We evaluated morbidity by diagnosis codes and medicine prescriptions before the PCOS diagnosis. The Charlson Index was significantly higher in patients with PCOS than in controls, which support an overall increased morbidity in PCOS. Increased morbidity in PCOS included cardiometabolic as well as autoimmune and psychiatric diagnoses. The mean age of the included study population was 30 years and our data support that medical diseases are prevalent also in young individuals diagnosed with PCOS. In a representative subgroup of patients with PCOS from our outpatient clinic, the presence of individual 
Table 4 Medical treatment in PCOS patients and controls (ever use). Data presented as $n$ (\%).

\begin{tabular}{|c|c|c|c|c|c|c|c|c|c|}
\hline \multirow[b]{2}{*}{ Characteristic } & \multirow[b]{2}{*}{ WHO ATC } & \multicolumn{2}{|c|}{$\begin{array}{c}\text { PCOS OUH } \\
(n=1217)\end{array}$} & \multirow[b]{2}{*}{$\boldsymbol{P}^{\mathrm{a}}$} & \multicolumn{2}{|c|}{$\begin{array}{l}\text { PCOS Denmark } \\
(n=19199)\end{array}$} & \multicolumn{2}{|c|}{$\begin{array}{l}\text { Controls } \\
(n=57483)\end{array}$} & \multirow[b]{2}{*}{$\boldsymbol{P}^{\mathrm{b}}$} \\
\hline & & $n$ & $\%$ & & $n$ & $\%$ & $n$ & $\%$ & \\
\hline \multicolumn{10}{|l|}{ Cardiometabolic } \\
\hline Metformin & A10BA02 & 92 & 7.5 & $<0.001$ & 2262 & 11.8 & 211 & 0.4 & $<0.001$ \\
\hline Glitazone & A10BG03, A10BD09 & 0 & 0 & 0.72 & 2 & 0.01 & 1 & 0.00 & 0.021 \\
\hline Insulin & A10A & 9 & 0.7 & 0.39 & 190 & 1.0 & 349 & 0.6 & $<0.001$ \\
\hline Other anti-diabetics & $\mathrm{A} 10 \mathrm{~B}$ & 93 & 7.6 & $<0.001$ & 2301 & 12.0 & 275 & 0.5 & $<0.001$ \\
\hline Anti-lipids & $\begin{array}{l}\text { C10AA, C10AB, C10AC, } \\
\text { C10AD, C10AX, C10BA02 }\end{array}$ & 17 & 1.4 & 0.83 & 283 & 1.5 & 419 & 0.7 & $<0.001$ \\
\hline Anti-hypertensives & $\mathrm{C} 03, \mathrm{C} 08, \mathrm{C} 09, \mathrm{CO} A \mathrm{~A}$ & 158 & 13.0 & 0.16 & 2774 & 14.5 & 3891 & 6.7 & $<0.001$ \\
\hline \multicolumn{10}{|c|}{ Thyroid, respiratory, CNS, inflammatory } \\
\hline $\begin{array}{l}\text { Thyroid hormones, } \\
\text { anti-thyroid treatment }\end{array}$ & H03А, Н03B & 32 & 2.6 & 0.87 & 520 & 2.7 & 508 & 0.9 & $<0.001$ \\
\hline Allergy medicine, steroid & R06A H02AB & 343 & 28.1 & 0.08 & 5876 & 30.6 & 13336 & 23.2 & $<0.001$ \\
\hline Asthma medicine & $\begin{array}{l}\text { R03AC, R03BB, R03DA, } \\
\text { R03BA, R03DC, R03AK }\end{array}$ & 237 & 19.5 & 0.85 & 3697 & 19.2 & 8101 & 14.1 & $<0.001$ \\
\hline Antidepressants & N06AA, N06AB, N06AX, N06A & 206 & 16.9 & 0.56 & 3128 & 16.3 & 5067 & 8.8 & $<0.001$ \\
\hline Neuroleptics & N05A & 51 & 4.2 & 0.18 & 969 & 5.0 & 1613 & 2.8 & $<0.001$ \\
\hline Anxiety medicine & $\begin{array}{l}\text { N06A, N05BA, N05BE, } \\
\text { N03AX16 }\end{array}$ & 270 & 22.2 & 0.97 & 4270 & 22.2 & 7277 & 12.6 & $<0.001$ \\
\hline Analgesic and migraine & N02 & 292 & 24.0 & 0.26 & 4885 & 25.4 & 8802 & 15.3 & 0.001 \\
\hline Antibiotic treatment & J01 & 1036 & 85.1 & 0.002 & 15695 & 81.7 & 41141 & 73.3 & $<0.001$ \\
\hline Smoke stop & $\begin{array}{l}\text { N07BA03, N07BA01 } \\
\text { N06AX12 }\end{array}$ & 11 & 0.9 & 0.80 & 188 & 1.0 & 486 & 0.9 & 0.086 \\
\hline \multicolumn{10}{|c|}{ Birth control, anti-androgens, and fertility } \\
\hline Oral contraceptives & G03AA, G03AB, & 607 & 49.8 & 0.28 & 9885 & 51.5 & 17057 & 29.7 & $<0.001$ \\
\hline 2nd generation OCP & $\begin{array}{l}\text { G03AA07, G03AA11, } \\
\text { G03AB03 }\end{array}$ & 181 & 14.8 & 0.26 & 3092 & 16.1 & 5414 & 9.4 & $<0.001$ \\
\hline 3rd generation OCP & $\begin{array}{l}\text { G03AA09, G03AA10, } \\
\text { G03AB06, G03AB05, } \\
\text { G02BB01 }\end{array}$ & 490 & 40.2 & 0.69 & 7841 & 40.8 & 13527 & 23.5 & $<0.001$ \\
\hline Drospirenone/ethinylestradiol & G03AA12 & 135 & 11.0 & 0.028 & 2549 & 13.2 & 2626 & 4.5 & $<0.001$ \\
\hline $\begin{array}{l}\text { Cyproterone acetate/ } \\
\text { ethinylestradiol }\end{array}$ & G03HB01 & 219 & 18.0 & 0.012 & 2943 & 15.3 & 1381 & 2.4 & $<0.001$ \\
\hline Gestagen & G03AC01, G03AC09, G03AC08 & 53 & 4.3 & 0.92 & 825 & 4.3 & 1488 & 2.6 & $<0.001$ \\
\hline IUD & G02BA, G02BA03 & 28 & 2.3 & 0.70 & 410 & 2.1 & 665 & 1.2 & $<0.001$ \\
\hline Spironolactone & C03DA01 & 10 & 0.8 & 0.07 & 279 & 1.5 & 70 & 0.1 & $<0.001$ \\
\hline Finasteride & G04CB & 0 & 0 & 0.80 & 1 & 0.0 & 45 & 0.1 & $<0.001$ \\
\hline Vaniqa & $\mathrm{D} 11 \mathrm{~A} \times 16$ & 0 & 0 & 0.47 & 8 & 0.0 & 0 & 0 & $<0.001$ \\
\hline Fertility treatment & G03GA G03GB, N04BC & 163 & 13.3 & $<0.001$ & 3318 & 17.3 & 1221 & 2.1 & $<0.001$ \\
\hline
\end{tabular}

OCP, oral contracpetive pills; IUD, intrauterine device. $P^{\mathrm{a}}$ Between PCOS OUH and PCOS Denmark, $P^{\mathrm{b}}$ Between PCOS Denmark and controls, $\chi^{2}$ test.

Rotterdam criteria was associated with obesity, but otherwise we found no association between the presence of individual Rotterdam criteria and cardiometabolic diagnoses.

Many different medical conditions have been associated with the diagnosis of PCOS (32). The risk of type 2 diabetes is currently estimated to be five to ten times increased in PCOS, whereas the risk of cardiovascular disease is debated $(3,9,13,33,34)$. The risk of cardiometabolic disease may be highly influenced by BMI, ethnicity, and the presence of different Rotterdam criteria, especially hyperandrogenism $(5,35)$, whereas high SHBG was a protective factor (34). In our cohort,
$1.5 \%$ patients were diagnosed with type 2 diabetes compared with $0.4 \%$ controls, which confirmed a five times increased risk of type 2 diabetes in PCOS. Given the register-based design, BMI data were not available in the PCOS Denmark group. The median BMI in PCOS OUH was $27.3 \mathrm{~kg} / \mathrm{m}^{2}$ and the relative risk of diabetes may be even higher in more obese study populations. Our findings of three times increased risk of dyslipidemia and hypertension in the PCOS Denmark cohort vs controls support clustering of cardiovascular risk factors in PCOS. Furthermore, the prescription of anti-lipids and antihypertensives was two times higher in patients with PCOS vs controls. Antidiabetic medicine including metformin is used to 
Table 5 Medical treatment in PCOS patients and controls (last year).

\begin{tabular}{|c|c|c|c|c|c|c|c|c|c|}
\hline \multirow[b]{2}{*}{ Characteristic } & \multirow[b]{2}{*}{ WHO ATC } & \multicolumn{2}{|c|}{$\begin{array}{l}\text { PCOS OUH } \\
(n=1217)\end{array}$} & \multirow[b]{2}{*}{$\boldsymbol{P}^{\mathrm{a}}$} & \multicolumn{2}{|c|}{$\begin{array}{c}\text { PCOS } \\
\text { Denmark } \\
(n=19 \text { 199) }\end{array}$} & \multicolumn{2}{|c|}{$\begin{array}{l}\text { Controls } \\
(n=57483)\end{array}$} & \multirow[b]{2}{*}{$\boldsymbol{P}^{\mathrm{b}}$} \\
\hline & & $n$ & $\%$ & & $n$ & $\%$ & $n$ & $\%$ & \\
\hline \multicolumn{10}{|l|}{ Cardiometabolic } \\
\hline Metformin & A10BA02 & 59 & 4.8 & $<0.001$ & 1795 & 9.4 & 146 & 0.3 & $<0.001$ \\
\hline Glitazone & A10BG03, A10BD09 & 0 & 0 & N/A & 0 & 0 & 1 & 0.0 & 0.084 \\
\hline Insulin & A10A & 8 & 0.6 & 0.46 & 165 & 0.9 & 321 & 0.6 & $<0.001$ \\
\hline Other anti-diabetics & $\mathrm{A} 10 \mathrm{~B}$ & 59 & 4.9 & $<0.001$ & 1826 & 9.5 & 193 & 0.3 & $<0.001$ \\
\hline Anti-lipids & $\begin{array}{l}\text { C10AA, C10AB, C10AC, } \\
\text { C10AD, C10AX, C10BA02 }\end{array}$ & 13 & 1.0 & 0.70 & 229 & 1.2 & 335 & 0.6 & $<0.001$ \\
\hline Anti-hypertensives & C03, C08, C09, C07A & 65 & 5.3 & 0.006 & 1431 & 7.5 & 1756 & 3.1 & $<0.001$ \\
\hline \multicolumn{10}{|c|}{ Thyroid, respiratory, CNS, inflammatory } \\
\hline $\begin{array}{l}\text { Thyroid hormones, anti-thyroid } \\
\text { treatment }\end{array}$ & H03A, H03B & 26 & 2.1 & 0.97 & 413 & 2.2 & 358 & 0.6 & $<0.001$ \\
\hline Allergy medicine, steroid & R06A H02AB & 108 & 8.9 & 0.78 & 1752 & 9.1 & 3601 & 6.3 & $<0.001$ \\
\hline Asthma medicine & $\begin{array}{l}\text { R03AC, R03BB, R03DA, } \\
\text { R03BA, R03DC, R03AK }\end{array}$ & 78 & 6.4 & 0.87 & 1253 & 6.5 & 2677 & 4.7 & $<0.001$ \\
\hline Anti-depressants & N06AA, N06AB, N06AX, N06A & 113 & 9.3 & 0.49 & 1672 & 8.7 & 2517 & 4.4 & $<0.001$ \\
\hline Neuroleptics & N05A & 24 & 2.0 & 0.36 & 458 & 2.4 & 738 & 1.3 & $<0.001$ \\
\hline Anxiety medicine & $\begin{array}{l}\text { N06A, N05BA, N05BE, } \\
\text { N03AX16 }\end{array}$ & 137 & 11.3 & 0.85 & 2127 & 11.1 & 3382 & 5.9 & $<0.001$ \\
\hline Analgesic and migraine & N02 & 110 & 9.0 & 0.27 & 1922 & 10.0 & 2939 & 5.1 & $<0.001$ \\
\hline Antibiotic treatment & J01 & 505 & 41.5 & 0.29 & 7671 & 40.0 & 16110 & 28.0 & $<0.001$ \\
\hline Smoke stop & $\begin{array}{l}\text { N07BA03, N07BA01 } \\
\text { N06AX12 }\end{array}$ & 2 & 0.2 & 0.31 & 64 & 0.3 & 151 & 0.3 & 0.10 \\
\hline \multicolumn{10}{|c|}{ Birth control, anti-androgens, and fertility } \\
\hline Oral contraceptives & G03AA, G03AB, & 227 & 18.7 & 0.55 & 3716 & 19.4 & 10031 & 17.5 & $<0.001$ \\
\hline 2nd generation OCP & $\begin{array}{l}\text { G03AA07, G03AA11, } \\
\text { G03AB03 }\end{array}$ & 38 & 3.1 & 0.40 & 692 & 3.6 & 2081 & 3.6 & 0.9 \\
\hline 3rd generation OCP & $\begin{array}{l}\text { G03AA09, G03AA10, } \\
\text { G03AB06, G03AB05, } \\
\text { G02BB01 }\end{array}$ & 138 & 11.3 & 0.63 & 2266 & 11.8 & 7207 & 12.5 & 0.006 \\
\hline Drospirenone/ethinylestradiol & G03AA12 & 65 & 5.3 & 0.81 & 996 & 5.2 & 1126 & 2.0 & $<0.001$ \\
\hline $\begin{array}{l}\text { Cyproterone acetate/ } \\
\text { ethinylestradiol }\end{array}$ & G03HB01 & 100 & 8.2 & 0.11 & 1347 & 7.0 & 533 & 0.9 & $<0.001$ \\
\hline Gestagen & G03AC01, G03AC09, G03AC08 & 13 & 1.0 & $<0.001$ & 825 & 4.3 & 457 & 0.8 & 0.01 \\
\hline IUD & G02BA, G02BA03 & 12 & 1.0 & 0.04 & 101 & 0.5 & 198 & 0.3 & $<0.001$ \\
\hline Spironolactone & C03DA01 & 7 & 0.6 & 0.11 & 201 & 1.1 & 36 & 0.2 & $<0.001$ \\
\hline Finasteride & G04CB & 0 & 0 & 0.80 & 1 & 0.01 & 10 & 0.02 & 0.22 \\
\hline Vaniqa & D11AX16 & 0 & 0 & 0.48 & 8 & 0.04 & 0 & 0 & $<0.001$ \\
\hline Fertility treatment & G03GA G03GB，N04BC & 36 & 3.0 & $<0.001$ & 1674 & 8.7 & 292 & 0.5 & $<0.001$ \\
\hline
\end{tabular}

OCP, oral contracpetive pills; IUD, intrauterine device. $P^{\mathrm{a}}$ Between PCOS OUH and PCOS Denmark, $P^{\mathrm{b}}$ Between PCOS Denmark and controls, $\chi^{2}$ test.

treat irregular menses, the metabolic syndrome, and infertility in PCOS, which could explain that the use of antidiabetics was up to 30 times increased in PCOS compared with controls. PCOS was associated with a two times increased risk of stroke and thrombosis, whereas the risk of other cardiac diseases was not increased. The presence of individual Rotterdam criteria was not associated with cardiometabolic outcome despite a more adverse metabolic risk profile in patients with PCO and irregular menses. It is possible that the inclusion of a young, relatively lean study population affected our results and further prospective studies are needed to determine whether the long-term metabolic risk is affected by PCOS phenotype. The number of cases diagnosed with thrombosis in PCOS OUH was limited and more data are needed on risk factors for thrombosis in patients with PCOS. Oral contraceptives are often used to treat hyperandrogenism and irregular menses in PCOS and five of six patients with thrombosis had been treated with oral contraceptives. Accordingly, the risk of venous thromboembolism was reported to be 1.5 -fold increased among women with PCOS not taking oral contraceptives and twofold increased among women with PCOS who were taking combined oral contraceptives (36). Furthermore, the risk 
Table 6 Rotterdam criteria in PCOS OUH population $(n=1217)$ and cardiometabolic diagnoses. Data presented as $n(\%)$.

\begin{tabular}{|c|c|c|}
\hline & \multicolumn{2}{|c|}{ Ultrasound $(n=1085)$} \\
\hline & PCO $(n=580)$ & Normal $(n=505)$ \\
\hline Obesity & $97(16.7) *$ & $56(11.1)$ \\
\hline Diabetes & $13(2.2)$ & $11(2.2)$ \\
\hline Type 1 diabetes & $3(0.5)$ & $3(0.6)$ \\
\hline Type 2 diabetes & $9(1.6)$ & $10(2.0)$ \\
\hline Gestational diabetes & $10(1.7)$ & $5(1.0)$ \\
\hline Dyslipidemia & $2(0.3)$ & $3(0.6)$ \\
\hline Hypertension & $6(1.0)$ & $6(1.2)$ \\
\hline Cardiovascular disease & $1(0.2)$ & 0 \\
\hline Mocardial infarction & $1(0.2)$ & $1(0.2)$ \\
\hline Transitory cerebral ischemia & $1(0.2)$ & $1(0.2)$ \\
\hline Stroke & $1(0.2)$ & $1(0.2)$ \\
\hline Thrombosis, lung embolism & $2(0.3)$ & $3(0.6)$ \\
\hline
\end{tabular}

\begin{tabular}{|c|c|}
\hline \multicolumn{2}{|c|}{ Hyperandrogenism $(n=1211)$} \\
\hline Yes $(n=1124)$ & No $(n=87)$ \\
\hline $149(13.3) *$ & $18(20.7)$ \\
\hline $27(2.4)$ & $2(2.3)$ \\
\hline $6(0.5)$ & $1(1.2)$ \\
\hline $21(1.9)$ & $2(2.3)$ \\
\hline $15(1.3)$ & $3(3.5)$ \\
\hline $3(0.3)$ & $2(2.3)$ \\
\hline $12(1.1)$ & $2(2.3)$ \\
\hline $2(0.2)$ & 0 \\
\hline $2(0.2)$ & 0 \\
\hline $1(0.1)$ & $1(1.1)$ \\
\hline $1(0.1)$ & $1(1.1)$ \\
\hline $4(0.4)$ * & $2(2.3)$ \\
\hline
\end{tabular}

\begin{tabular}{|c|c|}
\hline \multicolumn{2}{|c|}{ Menses $(n=1193)$} \\
\hline Irregular $(n=728)$ & Regular $(n=465)$ \\
\hline $122(16.7)^{\dagger}$ & $43(9.3)$ \\
\hline $19(2.6)$ & $10(2.2)$ \\
\hline $6(0.8)$ & $1(0.2)$ \\
\hline 14 (1.9) & $9(1.9)$ \\
\hline $13(1.8)$ & $5(1.1)$ \\
\hline $4(0.6)$ & $1(0.2)$ \\
\hline $9(1.2)$ & $5(1.1)$ \\
\hline $28(0.3)$ & 0 \\
\hline $1(0.1)$ & $1(0.2)$ \\
\hline $2(0.3)$ & 0 \\
\hline $2(0.3)$ & 0 \\
\hline $5(0.7)$ & $1(0.2)$ \\
\hline
\end{tabular}

${ }^{*} P<0.05$ and ${ }^{\dagger} P<0.001$ vs patients not fulfilling the individual Rotterdam criterion, $\chi^{2}$-test.

of thrombosis may be affected, which generation of oral contraceptive was used as 4th generation oral contraceptives containing drospirinone had the highest thromboembolic risk (37).

The overall presence of thyroid diseases was 3.6 times increased in PCOS vs controls. Correspondingly, prescriptions of thyroid medicine were three times increased in PCOS. These data are in agreement with clinical studies reporting that thyroid autoantibodies and autoimmune thyroiditis were more prevalent in patients with PCOS compared with controls $(16,38)$. These findings lead to a hypothesis of altered immune function in PCOS, which could be mediated by increased inflammatory status, unbalanced estrogen/progesterone secretion, or still unknown mechanisms (16). Similar mechanisms were suggested to affect respiratory health and to increase the risk of asthma in women with irregular menses (17). Our findings of significantly increased risk of asthma, however modest, underline the need for studies in patients with PCOS. Furthermore, the levels of various auto-antibodies were higher in PCOS vs controls (15) and the risk of rheumatologic or other autoimmune diseases may also be increased in PCOS (15).

Depression was 2.8 times more common in patients with PCOS vs controls and 1.9 times more patients than controls had prescriptions of antidepressants. The discrepancy between $1.4 \%$ patients diagnosed with depression and $16.3 \%$ patients treated with antidepressants could be explained by the fact that many patients are treated for depression by their own general practitioner and only hospital diagnoses are included in NPR. The present data support a moderately increased risk of depression in PCOS and are in agreement with recent studies $(2,20,39)$. In contrast, some studies reported an up to eightfold increased risk of depression in PCOS (18), but treatment with antidepressants is only indicated in moderate or severe and psychotherapy should be used in patients with milder symptoms. Based on the present data, we cannot conclude whether patients with PCOS are medically undertreated or have milder depression symptoms without need for medical treatment.

We found that the use of anxiety medicine was higher in patients with PCOS compared with controls. These results are supported by a recent register-based Taiwanese study reporting a hazard ratio of 1.4 for anxiety disorders in PCOS (39). Anxiety could be associated with depression and low quality of life in PCOS, but more studies are needed to test this hypothesis.

A novel finding of the present study was a two times higher risk of migraine/headache in PCOS vs controls and correspondingly a 1.7 times higher prescription rate of analgesic and migraine medicine. We are not aware of similar findings in previous studies and these results need to be reproduced in other cohorts of patients with PCOS. Testosterone treatment improved symptoms of migraine in pre- and postmenopausal women (40), whereas decreased dihydrotestosterone levels during treatment with finasteride improved migraine symptoms (41). Furthermore, increased use of analgesic medicine could be associated with the use of oral contraceptives, decreased quality of life in PCOS, or the presence of somatic diseases. The evaluation of the use of analgesic medicine is complicated by the fact that paracetamol and low dose NSAID can be bought over the counter in Denmark.

The prevalence of prior fractures was significantly lower in PCOS vs controls and support previous findings of normal or higher bone mineral density in PCOS and hirsute patients compared with controls (42). We did not 
evaluate the prevalence of osteoporosis in this study as the maximum age of included subjects was 60 years. Hyperandrogenemia (43), obesity (44), and hyperinsulinemia (45) may be bone protecting in PCOS. In contrast, vitamin D levels were lower in patients with PCOS and hirsutism vs controls (46) and the prevalence of vitamin D levels $<50 \mathrm{nmol} / \mathrm{l}$ in PCOS was 31-85\% (47). To our knowledge, only one previous study evaluated the fracture risk in women with PCOS (48). The fracture risk was unchanged in 25 postmenopausal women previously diagnosed with PCOS (48). Future prospective studies are therefore needed to determine fracture risk and possible predictors of fractures in pre-and postmenopausal women with PCOS.

Oral contraceptive treatment is the most widely applied treatment modality in PCOS (49). Oral contraceptives regulate menstrual cycles and SHBG levels increase, leading to decreased levels of free testosterone and decreased hirsutism scores (49). A nearly double prescription rate of oral contraceptives in PCOS was therefore not surprising. Furthermore, increased use of cycle regulating medicine, IUD, and antiandrogen treatment was expected in patients with PCOS. Fertility and pregnancy outcome was not the primary outcome of this study. Our data supported that the number of births was not reduced in patients with PCOS despite a significantly higher rate of diagnosed infertility and increased prescription of fertility treatment in PCOS. The inclusion of a relatively lean study population could explain some of our results as the number of births were reduced in obese study populations with PCOS (50). The observed fewer diagnoses of infertility and different distribution of number of births in PCOS OUH compared with PCOS Denmark may be explained by the exclusion of women with fertility plans from PCOS OUH.

The strength of this study is the inclusion of a large and well-characterized group of patients with PCOS, the ability to embed a clinical cohort into a national registerbased study, and the access to well validated register-based data with a long record of past drug exposures. Some limitations may however apply to this study design. No data were available on patients not attending the outpatient clinic. Patients not attending the clinic could differ from included patients. Many patients with PCOS are not seen at the hospital, but are treated by their general practitioner or a private gynecologist. Therefore, some women in the control group will have undiagnosed PCOS, which will lead to underestimation of morbidity and medicine use in PCOS. This study was not a cross sectional study on the prevalence of PCOS, but included patients diagnosed over a number of years. Therefore, there may be an influence of temporal trends in obesity and in the diagnostic effort and treatment patterns for other conditions, both in the PCOS patients and in control subjects. Patients with isolated hirsutism were included in both PCOS populations as the majority of patients with clinical and/or biochemical hyperandrogenism are diagnosed with PCOS and hyperandrogenism may be closely linked to metabolic disturbances in PCOS $(21,22,23)$. Patients were included from 1994 and onwards, whereas the Rotterdam criteria were introduced in 2003 (1). Especially, the criteria for PCO varied before 2003 and different criteria for PCOS may have been used by endocrinologists and gynecologists $(1,51)$. Furthermore, the definition of PCOS in adolescents is debated (52). The register-based design did not allow us to test the hypothesis that different definitions of PCOS affected study outcomes. We excluded patients and controls with a history of relevant endocrine conditions (1); however, the findings of the present study need to be confirmed in other study populations. Our cohort (PCOS OUH) differed from PCOS Denmark in terms of age, comorbidity, and prescription history. However, these differences were small and therefore unlikely to be a major source of bias in our findings. It is important to be aware that national registers, from which the PCOS Denmark cohort was drawn, do not contain information on BMI or on serum biochemistry. Hence, we are unable to determine if the higher prevalence of cardiometabolic disorders found in the PCOS cohort can be attributed to higher BMI alone or not. This does not detract from the finding that PCOS patients should be carefully screened for these diseases. Further, we were able to demonstrate that the PCOS Denmark cohort, though selected by ICD10 diagnosis codes alone, is reassuringly similar on key characteristics to our carefully clinically and biochemically evaluated PCOS OUH cohort. This is an important step forward as the validity of ICD10 coding for hirsutism in Denmark was previously not known.

In conclusion, morbidity and medicine use was significantly increased in a Danish population with PCOS. Our data confirm that medical diseases are often occurring also in young patients with PCOS. In this study, we found no association between the presence of individual Rotterdam criteria and cardiometabolic diagnoses in a well characterized group of patients with PCOS.

\footnotetext{
Declaration of interest
}

The authors declare that there is no conflict of interest that could be perceived as prejudicing the impartiality of the research reported. 
Funding

This research did not receive any specific grant from any funding agency in the public, commercial or not-for-profit sector.

\section{References}

1 Rotterdam ESHRE/ASRM-Sponsored PCOS Consensus Workshop Group. Revised 2003 consensus on diagnostic criteria and long-term health risks related to polycystic ovary syndrome. Fertility and Sterility 200481 19-25. (doi:10.1016/j.fertnstert.2003.10.004)

2 Conway G, Dewailly D, Diamanti-Kandarakis E, Escobar-Morreale HF, Franks S, Gambineri A, Kelestimur F, Macut D, Micic D, Pasquali R et al. The polycystic ovary syndrome: a position statement from the European Society of Endocrinology. European Journal Endocrinology 2014 4 1-29. (doi:10.1530/EJE-14-0253)

3 Glintborg D \& Andersen M. An update on the pathogenesis, inflammation, and metabolism in hirsutism and polycystic ovary syndrome. Gynecological Endocrinology 20104 281-296. (doi:10.3109/ 09513590903247873)

4 Azziz R. The evaluation and management of hirsutism. Obstetrics and Gynecology 20035 995-1007. (doi:10.1016/S0029-7844(02)02725-4)

5 Moghetti P, Tosi F, Bonin C, Di SD, Fiers T, Kaufman JM, Giagulli VA, Signori C, Zambotti F, Dall'Alda M et al. Divergences in insulin resistance between the different phenotypes of the polycystic ovary syndrome. Journal of Clinical Endocrinology and Metabolism 20134 E628-E637. (doi:10.1210/jc.2012-3908)

6 O'Reilly MW, Taylor AE, Crabtree NJ, Hughes BA, Capper F, Crowley RK, Stewart PM, Tomlinson JW \& Arlt W. Hyperandrogenemia predicts metabolic phenotype in polycystic ovary syndrome: the utility of serum androstenedione. Journal of Clinical Endocrinology and Metabolism 201499 1027-1036.

7 Ehrmann DA, Barnes RB, Rosenfield RL, Cavaghan MK \& Imperial J. Prevalence of impaired glucose tolerance and diabetes in women with polycystic ovary syndrome. Diabetes Care 19991 141-146. (doi:10.2337/diacare.22.1.141)

8 Glintborg D, Henriksen JE, Andersen M, Hagen C, Hangaard J, Rasmussen PE, Schousboe K \& Hermann AP. Prevalence of endocrine diseases and abnormal glucose tolerance tests in 340 Caucasian premenopausal women with hirsutism as the referral diagnosis. Fertility and Sterility 20046 1570-1579. (doi:10.1016/j.fertnstert.2004.06.040)

9 Tomlinson J, Millward A, Stenhouse E \& Pinkney J. Type 2 diabetes and cardiovascular disease in polycystic ovary syndrome: what are the risks and can they be reduced? Diabetic Medicine 20105 498-515. (doi:10.1111/j.1464-5491.2010.02994.x)

10 Pierpoint T, McKeigue PM, Isaacs AJ, Wild SH \& Jacobs HS. Mortality of women with polycystic ovary syndrome at long-term follow-up. Journal of Clinical Epidemiology 19987 581-586. (doi:10.1016/S08954356(98)00035-3)

11 Wild S, Pierpoint T, McKeigue P \& Jacobs H. Cardiovascular disease in women with polycystic ovary syndrome at long-term follow-up: a retrospective cohort study. Clinical Endocrinology 20005 595-600. (doi:10.1046/j.1365-2265.2000.01000.x)

12 Dahlgren E, Janson PO, Johansson S, Lapidus L \& Odén A. Polycystic ovary syndrome and risk for myocardial infarction. Evaluated from a risk factor model based on a prospective population study of women. Acta Obstetricia et Gynecologica Scandinavica 19928 599-604. (doi:10.3109/00016349209006227)

13 Mani H, Levy MJ, Davies MJ, Morris DH, Gray LJ, Bankart J, Blackledge H, Khunti K \& Howlett TA. Diabetes and cardiovascular events in women with polycystic ovary syndrome: a 20-year retrospective cohort study. Clinical Endocrinology 20136 926-934. (doi:10.1111/cen.12068)
14 Wild RA, Carmina E, Diamanti-Kandarakis E, Dokras A, EscobarMorreale HF, Futterweit W, Lobo R, Norman RJ, Talbott E \& Dumesic DA. Assessment of cardiovascular risk and prevention of cardiovascular disease in women with the polycystic ovary syndrome: a position statement by the Androgen Excess and Polycystic Ovary Syndrome (AE-PCOS) Society. Journal of Clinical Endocrinology and Metabolism 2010 5 2038-2049. (doi:10.1210/jc.2009-2724)

15 Hefler-Frischmuth K, Walch K, Huebl W, Baumuehlner K, Tempfer C \& Hefler L. Serologic markers of autoimmunity in women with polycystic ovary syndrome. Fertility and Sterility 20107 2291-2294. (doi:10.1016/j. fertnstert.2009.01.056)

16 Al-Saab R \& Haddad S. Detection of thyroid autoimmunity markers in euthyroid women with polycystic ovary syndrome: a case-control study from Syria. International Journal of Endocrinology and Metabolism 20143 e17954.

17 Macsali F, Svanes C, Bjorge L, Omenaas ER \& Gomez RF. Respiratory health in women: from menarche to menopause. Expert Review of Respiratory Medicine 20122 187-200. (doi:10.1586/ers.12.15)

18 Cinar N, Kizilarslanoglu MC, Harmanci A, Aksoy DY, Bozdag G, Demir B \& Yildiz BO. Depression, anxiety and cardiometabolic risk in polycystic ovary syndrome. Human Reproduction 201112 3339-3345. (doi:10.1093/humrep/der338)

19 Jones GL, Hall JM, Balen AH \& Ledger WL. Health-related quality of life measurement in women with polycystic ovary syndrome: a systematic review. Human Reproduction Update 20081 15-25.

20 Altinok ML, Glintborg D, Depont CR, Hallas J \& Andersen M. Prescription of antidepressants is increased in Danish patients with polycystic ovary syndrome and is associated with hyperandrogenism. A population-based cohort study. Clinical Endocrinology $2013 \mathbf{8 0}$ 884-889. (doi:10.1111/cen.12365)

21 Azziz R, Sanchez LA, Knochenhauer ES, Moran C, Lazenby J, Stephens KC, Taylor K \& Boots LR. Androgen excess in women: experience with over 1000 consecutive patients. Journal of Clinical Endocrinology and Metabolism 20042 453-462. (doi:10.1210/jc.2003-031122)

22 Escobar-Morreale HF, Carmina E, Dewailly D, Gambineri A, Kelestimur F, Moghetti P, Pugeat M, Qiao J, Wijeyaratne CN, Witchel SF et al. Epidemiology, diagnosis and management of hirsutism: a consensus statement by the Androgen Excess and Polycystic Ovary Syndrome Society. Human Reproduction Update 20122 146-170. (doi:10.1093/humupd/dmr042)

23 Azziz R, Carmina E, Dewailly D, Diamanti-Kandarakis E, EscobarMorreale HF, Futterweit W, Janssen OE, Legro RS, Norman RJ, Taylor AE et al. Positions statement: criteria for defining polycystic ovary syndrome as a predominantly hyperandrogenic syndrome: an Androgen Excess Society guideline. Journal of Clinical Endocrinology and Metabolism 200611 4237-4245. (doi:10.1210/jc.2006-0178)

24 Ferriman D \& Gallwey JD. Clinical assessment of body hair growth in women. Journal of Clinical Endocrinology 196121 1440-1447. (doi:10.1210/jcem-21-11-1440)

25 Lykkesfeldt G, Bennett P, Lykkesfeldt AE, Micic S, Moller S \& Svenstrup B. Abnormal androgen and oestrogen metabolism in men with steroid sulphatase deficiency and recessive X-linked ichthyosis. Clinical Endocrinology 19854 385-393. (doi:10.1111/j.1365-2265. 1985.tb01096.x)

26 Radziuk J. Insulin sensitivity and its measurement: structural commonalities among the methods. Journal of Clinical Endocrinology and Metabolism 200012 4426-4433.

27 Lynge E, Sandegaard JL \& Rebolj M. The Danish National Patient Register. Scandinavian Journal of Public Health 201139 (7 Suppl) 30-33. (doi:10.1177/1403494811401482)

28 Nader S. Hyperandrogenism during puberty in the development of polycystic ovary syndrome. Fertility and Sterility $2013139-42$. (doi:10.1016/j.fertnstert.2013.03.013)

29 Forman MR, Mangini LD, Thelus-Jean R \& Hayward MD. Life-course origins of the ages at menarche and menopause. Adolescent Health, Medicine and Therapeutics 20134 1-21. (doi:10.2147/AHMT.S15946) 
30 Charlson ME, Pompei P, Ales KL \& MacKenzie CR. A new method of classifying prognostic comorbidity in longitudinal studies: development and validation. Journal of Chronic Diseases 19875 373-383. (doi:10.1016/0021-9681(87)90171-8)

31 Quan H, Sundararajan V, Halfon P, Fong A, Burnand B, Luthi JC, Saunders LD, Beck CA, Feasby TE \& Ghali WA. Coding algorithms for defining comorbidities in ICD-9-CM and ICD-10 administrative data. Medical Care 200511 1130-1139. (doi:10.1097/01.mlr.0000182534. 19832.83)

32 Morgan CL, Jenkins-Jones S, Currie CJ \& Rees DA. Evaluation of adverse outcome in young women with polycystic ovary syndrome versus matched, reference controls: a retrospective, observational study. Journal of Clinical Endocrinology and Metabolism 20129 3251-3260. (doi:10.1210/jc.2012-1690)

33 Bajuk SK, Jensterle SM \& Pfeifer M. Cardiovascular risk and subclinical cardiovascular disease in polycystic ovary syndrome. Frontiers of Hormone Research 201340 64-82. (doi:10.1159/000341838)

34 Gambineri A, Patton L, Altieri P, Pagotto U, Pizzi C, Manzoli L \& Pasquali R. Polycystic ovary syndrome is a risk factor for type 2 diabetes: results from a long-term prospective study. Diabetes 20129 2369-2374. (doi:10.2337/db11-1360)

35 Glintborg D, Mumm H, Hougaard D, Ravn P \& Andersen M. Ethnic differences in Rotterdam criteria and metabolic risk factors in a multiethnic group of women with PCOS studied in Denmark. Clinical Endocrinology 20106 732-738. (doi:10.1111/j.1365-2265.2010.03873.x)

36 Bird ST, Hartzema AG, Brophy JM, Etminan M \& Delaney JA. Risk of venous thromboembolism in women with polycystic ovary syndrome: a population-based matched cohort analysis. CMAJ: Canadian Medical Association Journal 20132 E115-E120. (doi:10.1503/cmaj.120677)

37 Gronich N, Lavi I \& Rennert G. Higher risk of venous thrombosis associated with drospirenone-containing oral contraceptives: a population-based cohort study. CMAJ: Canadian Medical Association Journal 201118 E1319-E1325. (doi:10.1503/cmaj.110463)

38 Novais JD, Benetti-Pinto CL, Garmes HM, Menezes JR \& Juliato CR. Polycystic ovary syndrome and chronic autoimmune thyroiditis. Gynecological Endocrinology 201431 1-4. (doi:10.3109/09513590.2014. 958990)

39 Hung JH, Hu LY, Tsai SJ, Yang AC, Huang MW, Chen PM, Wang SL, Lu T $\&$ Shen CC. Risk of psychiatric disorders following polycystic ovary syndrome: a nationwide population-based cohort study. PLOS ONE 20145 e97041. (doi:10.1371/journal.pone.0097041)

40 Glaser R, Dimitrakakis C, Trimble N \& Martin V. Testosterone pellet implants and migraine headaches: a pilot study. Maturitas 20124 385-388. (doi:10.1016/j.maturitas.2012.01.006)

41 Check JH \& Cohen R. Dihydrotestosterone may contribute to the development of migraine headaches. Clinical and Experimental Obstetrics \& Gynecology 20132 217-218.
42 Glintborg D, Hermann A \& Andersen M. Bone mineral density and vitamin D in PCOS and hirsutism. Expert Review of Endocrinology \& Metabolism 20135 449-459. (doi:10.1586/17446651.2013.827384)

43 Gregoriou O, Kouskouni E, Bakas P, Konidaris S, Papadias K, Kalovidouris A \& Creatsas G. Bone mineral density in women with idiopathic hirsutism. Gynecological Endocrinology 20005 364-368. (doi:10.3109/09513590009167704)

44 Reid IR, Plank LD \& Evans MC. Fat mass is an important determinant of whole body bone density in premenopausal women but not in men. Journal of Clinical Endocrinology and Metabolism 19923 779-782. (doi:10.1210/jcem.75.3.1517366)

45 Abrahamsen B, Rohold A, Henriksen JE \& Beck-Nielsen H. Correlations between insulin sensitivity and bone mineral density in non-diabetic men. Diabetic Medicine 20002 124-129. (doi:10.1046/j.1464-5491. 2000.00234.x)

46 Glintborg D, Andersen M, Hagen C \& Hermann AP. Higher bone mineral density in Caucasian, hirsute patients of reproductive age. Positive correlation of testosterone levels with bone mineral density in hirsutism. Clinical Endocrinology 20056 683-691. (doi:10.1111/j.13652265.2005.02280.x)

47 Thomson RL, Spedding S, Brinkworth GD, Noakes M \& Buckley JD. Seasonal effects on vitamin D status influence outcomes of lifestyle intervention in overweight and obese women with polycystic ovary syndrome. Fertility and Sterility 20136 1779-1785. (doi:10.1016/j. fertnstert.2012.12.042)

48 Schmidt J, Dahlgren E, Brannstrom M \& Landin-Wilhelmsen K. Body composition, bone mineral density and fractures in late postmenopausal women with polycystic ovary syndrome - a long-term follow-up study. Clinical Endocrinology 20122 207-214. (doi:10.1111/j.1365-2265. 2012.04378.x)

49 Sirmans SM \& Pate KA. Epidemiology, diagnosis, and management of polycystic ovary syndrome. Clinical Epidemiology 20136 1-13. (doi:10.2147/CLEP.S37559)

50 De Frene V, Vansteelandt S, T'Sjoen G, Gerris J, Somers S, Vercruysse L $\&$ De Sutter P. A retrospective study of the pregnancy, delivery and neonatal outcome in overweight versus normal weight women with polycystic ovary syndrome. Human Reproduction 201410 2333-2338. (doi:10.1093/humrep/deu154)

51 Cussons AJ, Stuckey BG, Walsh JP, Burke V \& Norman RJ. Polycystic ovarian syndrome: marked differences between endocrinologists and gynaecologists in diagnosis and management. Clinical Endocrinology 20053 289-295. (doi:10.1111/j.1365-2265.2004.02208.x)

52 Legro RS, Arslanian SA, Ehrmann DA, Hoeger KM, Murad MH, Pasquali R \& Welt CK. Diagnosis and treatment of polycystic ovary syndrome: an Endocrine Society clinical practice guideline. Journal of Clinical Endocrinology and Metabolism 201312 4565-4592. (doi:10.1210/ jc.2013-2350)

Received 12 December 2014

Revised version received 2 February 2015

Accepted 5 February 2015 